\section{Vergütungsregeln in der Schmerztherapie}

Die Auslegung einer Gebührenordnungsposition (GOP) orientiert sich in erster Linie an dem Wortlaut der Leistungslegende, bei bestimmten Konstellationen jedoch nicht ausschließlich. Bei Unklarheiten der Leistungslegende können auch andere Auslegungsregelungen wie die systematische Auslegung und die historische Auslegung zur Anwendung kommen. Die systematische und die historische Auslegung legen es zwingend nahe, dass für die Abrechnung der GOP 30702 (Zusatzpauschale für die schmerztherapeutische Versorgung gemäß der Qualitätssicherungsvereinbarung zur schmerztherapeutischen Versorgung chronisch schmerzkranker Patienten nach § 135 Abs. 2 SGB V) eine Dauer von mindestens 60 Minuten vorauszusetzen und in diesem Zeitraum nicht zusätzlich die GOP 30708 (Beratung und Erörterung und/oder Abklärung im Rahmen der Schmerztherapie) abzurechnen ist.

Sozialgericht München, Urteil vom 21.07.2017 - S 38 KA 1012/15

\section{Anästhesist hat Erfolg bei sachlich- rechnerischer Richtigstellung}

Die Berechnung der EBM-Ziffer 30731 setzt - anders als die der früheren EBMNr. 443 - nicht voraus, dass eine fachspezifische Dokumentation (nach Nr. 5 der Präambel 5.1 zu den anästhesiologischen Gebührenordnungspositionen) erstellt worden ist. Eine Kassenärztliche Vereinigung kann aus der fehlenden Vorlage der Dokumentation bestimmter vertragsärztlicher Leistungen im Rahmen der nachgehenden Honorarprüfung nur dann den Schluss ziehen, die Leistung sei nicht erbracht worden, wenn sie dem Vertragsarzt zuvor unmissverständlich mitgeteilt hat, welche Mitwirkungshandlungen von ihm erwartet werden. Dass die Abrechnung des Klägers, eines vertragsärztlich tätigen Facharztes für Anästhesiologie, hinsichtlich der Abrechnung von Leistungen nach Ziffer 30731 EBM fehlerhaft gewesen ist, kann vor diesem Hintergrund nicht festgestellt werden.

Landessozialgericht Niedersachsen-Bremen, Urteil vom 06.09.2017 - L 3 KA 108/14 scheidung über die Zulassung und Genehmigung der Verlegung einer Arztund einer Psychotherapeutenstelle beschränkt.

Das Sozialgericht Hamburg (SG) wies die Klage ab und schloss sich damit der Ansicht des Beklagten an (Urteil vom 28.09.2016 - Az.: S 27 KA 39/16). Das SG begründete seine Entscheidung damit, dass der Anspruch der Klägerin nicht durch $\$ 24$ Abs 7 Satz 2 Ärzte-Zulassungsverordnung (ZV) (siehe Kasten) gestützt werde, der seit 2015 die Verlegung von Sitzen angestellter Ärzte zwischen verschiedenen Medizinischen Versorgungszentren ermögliche.

Über eine Sprungrevision direkt zum BSG, unter Umgehung der Berufungsin$\operatorname{stanz}$ zum Landessozialgericht (LSG), machte die Klägerin geltend, dass eine gesetzliche Regelung, die der Zulassung des MVZ entgegenstehen könnte, nicht existiere. Die ablehnende Haltung des Beklagten werde nur von der Erwägung getragen, dass den Zentren keine Gestaltungsmöglichkeiten eröffnet werden dürften, die den Vertragsärzten nicht zur Verfügung stünden. Diese Bewertung reiche für eine Versagung ihres $\mathrm{Zu}$ lassungsanspruchs nicht aus, so die Klägerin.

\section{Das Urteil}

Die Revision der klagenden $\mathrm{GmbH}$ hatte jedoch keinen Erfolg. Der beklagte Berufungsausschuss hat nach dem Urteil des BSG die Anträge der Klägerin auf Zulassung und Genehmigung der Anstellung einer Ärztin und einer Psychotherapeutin abgelehnt.

Nach der Auffassung des BSG kann durch die Verlegung von Arztanstellungen auf der Grundlage des $\$ 24$ Abs 7 Satz 2 Ärzte-Zulassungsverordnung kein neuer Zulassungsstatus begründet werden. Die durch das BSG im Juli 2015 eingeführte Vorschrift ermöglicht zwar die Verlegung von Arztanstellungen von einem MVZ in ein anderes MVZ desselben Betreibers oder einer anderen Betreibergesellschaft mit denselben Gesellschaftern. Nach dem Wortlaut, der systematischen Stellung und der Entstehungsgeschichte kann die Norm, so das BSG, allerdings keine Grundlage für die Schaffung eines neuen, zusätzlichen $\mathrm{Zu}$ lassungsstatus sein. Deshalb ist es auch ohne Bedeutung, dass die von der Klägerin gewünschte neue Zulassung keine Überversorgung zur Folge hätte und somit bedarfsplanungsrechtlich neutral wäre.

Auch eine entsprechende Anwendung des $\$ 103$ Abs 4a Satz 1 SGB V trägt nach dem Urteil des BSG das Begehren der Klägerin nicht. Die Klägerin verzichtet nämlich nicht im Sinne der Vorschrift auf ihre Zulassung, sodass eine Tätigkeit in einem MVZ im Anstellungsverhältnis nicht möglich ist.

$\$ 103$ Abs 4a Satz 1 SGB V lautet: Verzichtet ein Vertragsarzt in einem Planungsbereich, für den Zulassungsbeschränkungen angeordnet sind, auf seine Zulassung, um in einem medizinischen Versorgungszentrum tätig zu werden, so hat der Zulassungsausschuss die Anstellung zu genehmigen, wenn Gründe der vertragsärztlichen Versorgung dem nicht entgegenstehen; eine Fortführung der Praxis nach Absatz 4 ist nicht möglich.

\section{Folge für die Zukunft des MVZ}

Das Urteil des BSG fügt sich in den MVZ-kritischen Kontext der BSGRechtsprechung ein, die auf eine Restriktion der gesetzlichen Intentionen für Medizinische Versorgungszentren abzielen. Man mag bedauern, dass der Pluralität der Bedarfe die Akzeptanz durch die Rechtsprechung erneut versagt wird. Wo der Gewinn für die Versorgung oder auch nur einzelne Akteure liegen könnte, der durch derartige Restriktionen bedingt ist, ist kaum nachzuvollziehen. Es bleibt abzuwarten, ob solch eine restriktive Auffassung unter den kritischen Augen des Gesetzgebers prolongiert oder korrigiert wird.

BSG Urteil vom 11.10.2017Az.: B 6 KA 38/16R

\section{Literatur beim Verfasser}

\section{Arno Zurstraßen M.A}

Rechtsanwalt und Mediator im

Gesundheitswesen, Supervision

Fachanwalt für Medizinrecht

und Sozialrecht

Aachener Straße 197-199, 50931 Köln

contact@arztundrecht.de

www.arztundrecht.de 